\title{
Self-assembled Amphiphilic Block Copolymers
}

\author{
H. Friedrich*, B. McKenzie**, P.H.H. Bomans*, Z. Deng*, F. Nudelman*, S.J. Holder**, G. de \\ With*, and N.A.J.M. Sommerdijk* \\ * Laboratory of Materials \& Interface Chemistry and Soft Matter CryoTEM Research Unit, \\ Eindhoven University of Technology, Postbus 513, 5600 MB Eindhoven, The Netherlands \\ ** Functional Materials Group, School of Physical Science, University of Kent at Canterbury, \\ Canterbury, Kent, CT2 7NZ, United Kingdom
}

Amphiphilic block copolymers expose the potential to self-assemble in aqueous media providing an ever expanding library of aggregate morphologies. In contrast to top down structuring approaches, self-assembly on the nanoscale has the capacity for an economical manufacture of polymeric and hybrid materials with well defined morphologies and properties. Our work examines the effects of polymer composition and preparation conditions on the aggregate structure of amphiphiles in their aqueous environment using cryogenic transmission electron microscopy (cryo-TEM) and cryo electron tomography (cryo-ET).

In the present study solutions of Poly (ethylene oxide) -b- Poly (octadecyl methacrylate) (PEOPODMA) in tetrahydrofuran (THF) were prepared at $35{ }^{\circ} \mathrm{C}$ followed by slow addition of water. The milky suspension was subsequently dialyzed twice in demineralized water for a total of 12 hours. Samples were stored and vitrified at $4{ }^{\circ} \mathrm{C}$ using a Vitrobot ${ }^{\circledR}$. Cryo-TEM and cryo-ET were performed on the TU/e cryoTITAN microscope.

As recently shown for related diblock polymers [1] also for PEO-PODMA spherical nanoaggregates with a bicontinuous internal structure were formed (Fig. 1) [2]. As we intend to use the internal aqueous compartments as selective sites where mineralization takes place a detailed analysis of the internal structure, in particular compartment size, morphology, connectivity and accessibility are key. Cryo-ET acquisition $\left(0^{\circ}\right.$ to $-64^{\circ} ; 0$ to $+64^{\circ}$ at $2^{\circ}$ increments $)$ was optimized by limiting the accumulated dose to less than $70 \mathrm{e}^{-} / \mathrm{A}^{2}$ and adjusting the exposure time with tilt $\left(\mathrm{I} / \mathrm{I}_{0}=1.6\right)$. To our surprise datasets acquired at constant exposure times $\left(1 \mathrm{~s}=1 \mathrm{e} / \mathrm{A}^{2}\right)$ displayed a much more pronounced shrinking of the spherical aggregates in their vitreous matrix. Datasets without shrinkage were alignment and reconstructed in Inspect3D and IMOD. An example of the results using different reconstruction algorithms (WB, SIRT, ART) are presented in Fig. 2. The visually smoothest and clearest results were obtained using the SIRT algorithm with 5 to 10 iterations as compared to 20 iterations which are commonly used in materials science ET. Fewer iteration steps, thus effectively combining reconstruction and filtering, seemed to be favorable on account of the low signal to noise ratio of the cryo-ET images. The challenge ahead lies in segmenting and quantifying the reconstructions to develop a comprehensive understanding of the self assembly process. Cryo-TEM and cryo-ET provide detailed insights into the structure of soft materials in their aqueous environment and are thus indispensable techniques within the field of chemistry.

[1] Parry, A.L.; Bomans, P.H.H.; Holder, S.J.; Sommerdijk, N.A.J.M.; Biagini, S. C. G. Angew. Chem. Int. Ed. 47 (2008) 8859.

[2] McKenzie, B; Nudelman, F.; Bomans, P.H.H.; Holder, S.J.; Sommerdijk, N.A.J.M. in preparation 

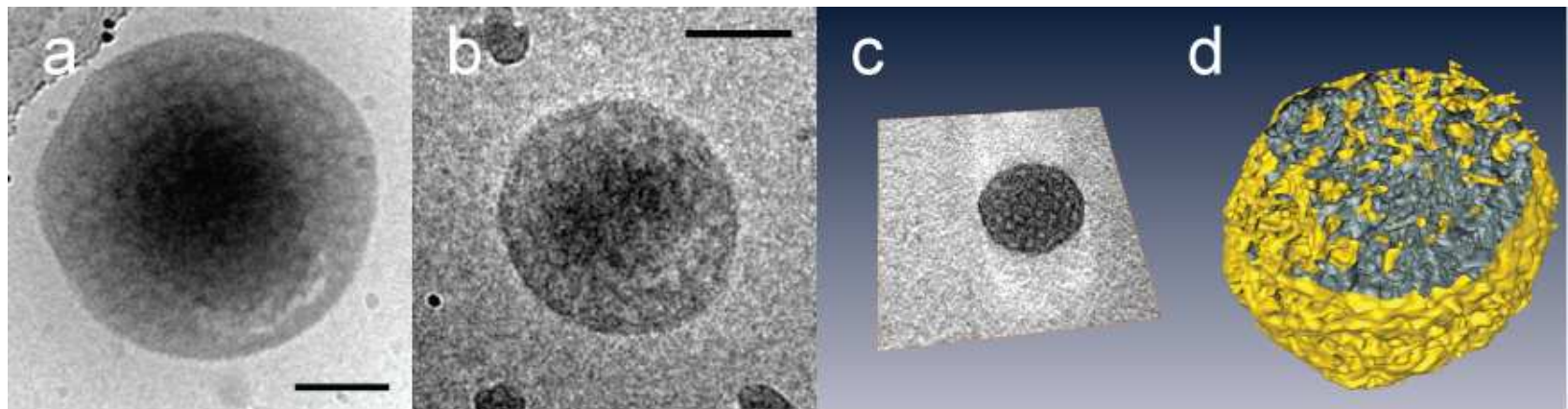

FIG. 1. Spherical PEO-PODMA aggregate with bicontinuous internal structure. (a,b) cryo-TEM images, (c) numerical cross-section through reconstruction of particle in (b), (d) isosurface visualization ('cut open') showing bicontinuous internal structure. Scale bars are 100nm.
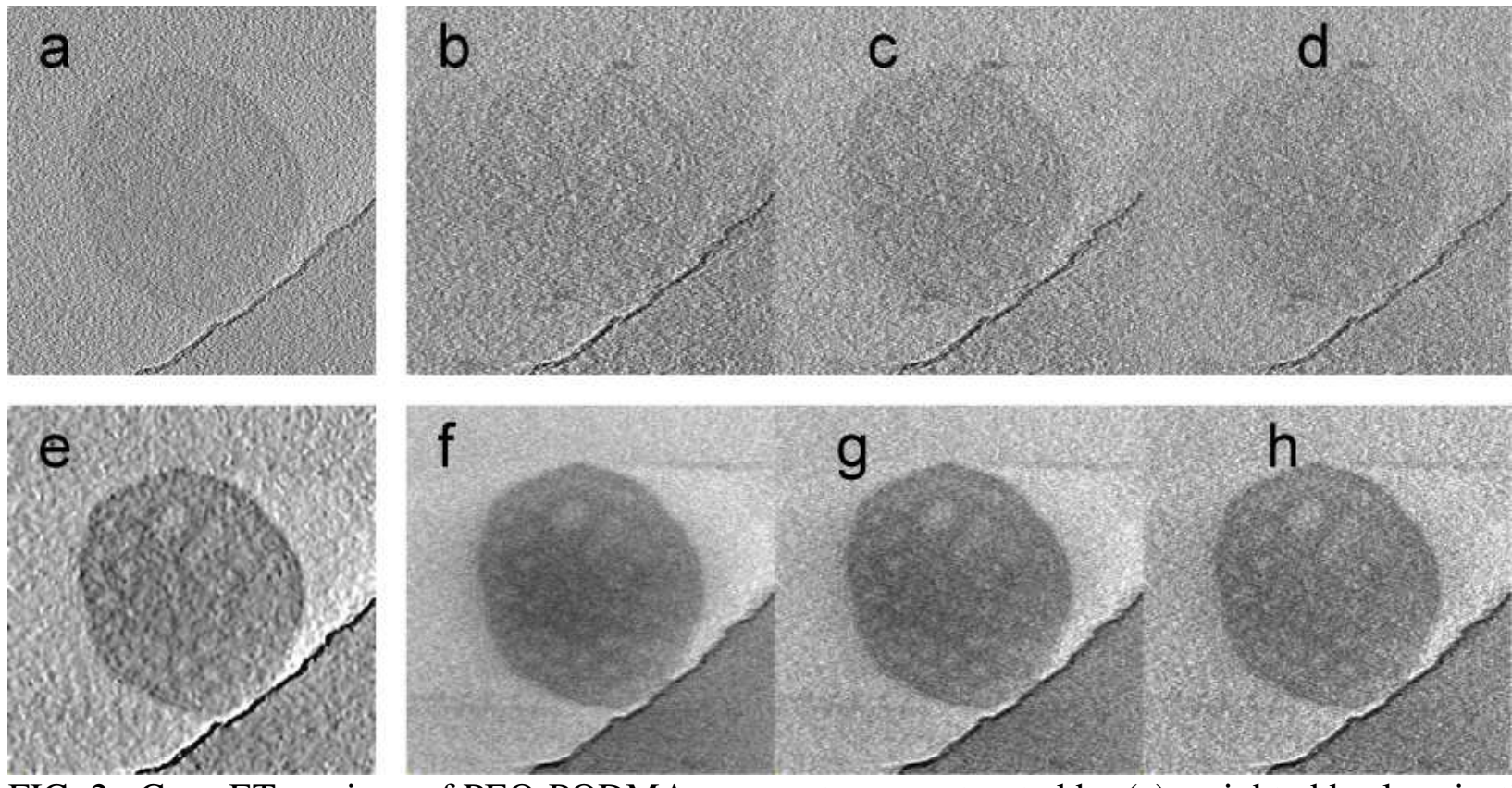

FIG. 2. Cryo-ET sections of PEO-PODMA aggregate reconstructed by (a) weighted backprojection; (b-d) ART with 3,5,10 iterations, respectively; (e) weighted backprojection and denoising by nonlinear anisotropic diffucion $(\mathrm{k}=64, \mathrm{i}=0.25)$; and (f-h) SIRT with 5,10,20 iterations respectively. 